\title{
Comparative effects of histone deacetylase inhibitors on p53 target gene expression, cell cycle and apoptosis in MCF-7 breast cancer cells
}

\author{
ANDREW KEKAPA'A KNUTSON ${ }^{1}$, JENNIFER WELSH ${ }^{1}$, TRAVIS TAYLOR ${ }^{2}$, SOMDUTTA ROY $^{3}$, \\ WEI-LIN WINNIE WANG ${ }^{1,4}$ and MARTIN TENNISWOOD ${ }^{1,4}$
}

\begin{abstract}
${ }^{1}$ Department of Biological Sciences, University of Notre Dame, Notre Dame, IN 46556; ${ }^{2}$ Department of Biological Sciences, Indiana University South Bend, South Bend, IN 46634; ${ }^{3}$ Department of Pathology, University of California San Francisco, San Francisco, CA 94143; ${ }^{4}$ Cancer Research Center and Department of Biomedical Sciences,
\end{abstract} School of Public Health, University at Albany, Albany, NY 12144, USA

Received October 6, 2011; Accepted November 10, 2011

DOI: $10.3892 / o r .2011 .1590$

\begin{abstract}
Histone deacetylase inhibitors are currently being evaluated for their therapeutic potential and have shown considerable promise as adjuvant therapies for a number of cancers. This study compared the effects of 2 hydroxamic acid based inhibitors, CG-1521 and SAHA, on gene expression, cell cycle and cell death in MCF-7 human breast cancer cells. Both compounds show a dose- and time-dependent effect on cell number (evaluated using crystal violet), however CG-1521 exerts its effects significantly earlier than SAHA, and CG-1521 induces apoptosis (assessed by Apo-BrdU staining and flow cytometry) more rapidly than SAHA. qPCR of cell cycle regulatory and apoptotic genes shows that CG-1521 and SAHA modulate similar cohorts of p53-responsive genes, however, the levels of induction and the timing of the induction differs significantly between the 2 inhibitors. In particular SAHA downregulates cell cycle-associated genes that modulate the $\mathrm{G}_{1} / \mathrm{S}$ transition (including cyclin D1 and cdc25a) and the $\mathrm{G}_{2} / \mathrm{M}$ transition [cyclin B1, Plk1, Stk6 (serine-threonine kinase 6, Aurora kinase A) and Kntc2] more significantly than CG-1521. In contrast, CG-1521 significantly induces the expression of several p53 target genes associated with apoptosis including Bnip3/Bnip3L, p21/p21B and Gdf15. The differential levels of gene induction provide molecular evidence of both cell cycle arrest and apoptosis, and suggest a molecular mechanism that explains the difference in the biological effects of the 2 histone deacetylase inhibitors.
\end{abstract}

Correspondence to: Dr Martin Tenniswood, Cancer Research Center and Department of Biomedical Sciences, School of Public Health, University at Albany, Albany, NY 12144, USA

E-mail: mtenniswood@albany.edu

Key words: breast cancer, protein acetylation, histone deacetylase inhibitors, mitotic catastrophe, cell death, gene expression

\section{Introduction}

Histone deacetylases (HDACs) cooperate with histone acetyltransferases (HATs) to regulate the acetylation status of nuclear histones, transcription factors and other cellular proteins to regulate a variety of cellular processes including cell division and gene expression and cell death (1-3). Based on their homology to yeast enzymes, phylogenetic analysis and subcellular localization, HDACs can be subdivided into 2 families: the classical HDAC family and the sirtuins. The classical family of HDACs are $\mathrm{Zn}^{2+}$ dependent enzymes: class I HDACs (HDACs 1, 2, 3, and 8) share homology to the yeast HDAC Rpd 3 and are localized in the nucleus; class II HDACs (HDACs 4, 5, 6, 7, 9 and 10) are related to yeast Hda1 and shuttle between the cytosol and nucleus $(4,5)$. Sirtuins, also referred to as class III HDACs, are $\mathrm{NAD}^{+}$dependent and are classified based on their homology to the yeast Sir2 (silent information regulator 2) (6). Based on their chemical structure, HDAC inhibitors (HDACi) can be divided into several classes including hydroxamic acid derivatives, benzamides, short chain fatty acids, cyclic tetrapeptides, and electrophilic ketones. Hydroxamic acid derivatives, including trichostatin A (TSA), suberoylanilide hydroxamic acid (SAHA), and CG-1521 (7-phenyl-2,4,6-hepta-trienoic hydroxamic acid) inhibit HDACs by coordinating the catalytic site $\mathrm{Zn}^{2+}$ (7). In contrast, effective inhibitors of the $\mathrm{NAD}^{+}$-dependent sirtuins have only been recently developed (8), and their potential utility in cancer therapies has been demonstrated recently (9). Previous studies from this laboratory have compared the effects of TSA and CG-1521 in p53 wild type LNCaP prostate cancer cell lines $(10,11)$. These studies established that the 2 hydroxamic acid-based inhibitors, target different lysine residues in p53, stimulate the assembly of different transcription complexes, and elicit different biological responses, even though they are both regarded as pan-HDAC inhibitors (12).

In this study we compare the effects of CG-1521 and SAHA on the induction of apoptosis in p53 wild type MCF-7 breast cancer cells to determine whether the effects of these HDAC inhibitors are cell-type specific. 


\section{Materials and methods}

Cell culture. MCF-7 human breast cancer cells were obtained from the American Type Culture Collection (Rockville, MD, USA) and cultured in $\alpha$-MEM media (Gibco, Grand Island, NY, USA) with 5\% fetal bovine serum (Gibco) and Pen/Strep. Cells were passaged every 4 or 5 days.

Crystal violet assay. Cells were seeded at $2 \times 10^{4}$ cells/well in 24-well plates. CG-1521 (Errant Gene Therapeutics, Chicago, IL, USA), SAHA (Errant Gene Therapeutics), or DMSO treatments were initiated $24 \mathrm{~h}$ after seeding and crystal violet growth assays were performed according to manufacturer's directions at 0-96 h (Fisher Scientific, Pittsburgh, PA).

Flow cytometry. Cells were seeded in 150x $25 \mathrm{~mm}$ culture dishes at a density of $1 \times 10^{6}$ cells/dish and treated with $7.5 \mu \mathrm{M}$ CG-1521 or $7.5 \mu \mathrm{M}$ SAHA dissolved in DMSO. Cells treated with vehicle (EtOH and DMSO) served as the negative control. For cell cycle analysis, cells were treated for $48 \mathrm{~h}$ and harvested by trypsinization and fixed with $90 \%$ ethanol overnight at $-20^{\circ} \mathrm{C}$. Permeabilized cells were stained with $5 \mu \mathrm{g} / \mathrm{ml}$ propidium iodide (Sigma-Aldrich) in the presence of $0.015 \mathrm{U} / \mathrm{ml}$ RNase (Roche Applied Science, Indianapolis, IN) in PBS with $0.1 \%$ Triton X-100 for $20 \mathrm{~min}$ at room temperature. Samples were analyzed by the Coulter Epics XL cytometer (Coulter, Miami, FL, USA) within $4 \mathrm{~h}$ of labeling. A minimum of 10,000 events were analyzed for each experimental condition and cell cycle kinetics were analyzed using the Multiplus AV software (Phoenix Flow Systems, San Diego, CA, USA). Apoptotic induction was quantified utilizing the Apo-BrdU TUNEL assay. Cells were fixed in $4 \%$ formaldehyde, washed with PBS $/ 0.2 \%$ BSA twice and permeabilized with $70 \%$ ethanol overnight. DNA strand breaks were labeled with bromo-deoxyuridine (BrdU) by terminal deoxynucleotidyl transferase according to the Apo-BrdU detection kit (Roche Diagnostics). Cells were incubated in the dark with FITC conjugated anti-BrdU monoclonal antibody (Phoenix Flow Systems). Total DNA was counterstained with propidium iodide and at least 10,000 cells per treatment were analyzed. Apoptosis was measured using the Coulter Epics XL cytometer.

Reverse transcriptase PCR. MCF-7 cells were seeded in $100 \times 25 \mathrm{~mm}$ culture dishes at a density of $5 \times 10^{5}$ cells/dish Cells were treated with $7.5 \mu \mathrm{M}$ CG-1521 or $7.5 \mu \mathrm{M}$ SAHA $24 \mathrm{~h}$ after seeding and RNA was extracted using the Qiagen RNeasy ${ }^{\mathrm{TM}}$ mini kit (Qiagen, Valencia, CA). RNA concentration and purity was determined and PCR was performed using $1.5 \mu \mathrm{g}$ of RNA in $100 \mu \mathrm{l}$ reaction with the TaqMan reverse transcription reagents (Applied Biosystems, Foster City, CA). The reaction was incubated for $10 \mathrm{~min}$ at $25^{\circ} \mathrm{C}, 1 \mathrm{~h}$ at $37^{\circ} \mathrm{C}$, and $5 \mathrm{~min}$ at $95^{\circ} \mathrm{C}$ and kept at $4^{\circ} \mathrm{C}$. Each cDNA synthesis was duplicated from 3 independent sets of RNA extractions.

Quantitative real-time PCR. Gene induction was measured using the SYBR-Green chemistry on an ABI Prism 7700 sequence detector (Applied Biosystems). Reactions were carried out according to the manufacturer's protocol. Briefly, samples were incubated for $2 \mathrm{~min}$ at $50^{\circ} \mathrm{C}, 10 \mathrm{~min}$ at $95^{\circ} \mathrm{C}$, $15 \mathrm{sec}$ at $95^{\circ} \mathrm{C}$, and $1 \mathrm{~min}$ at $60^{\circ} \mathrm{C}$ for 40 cycles. Primers were

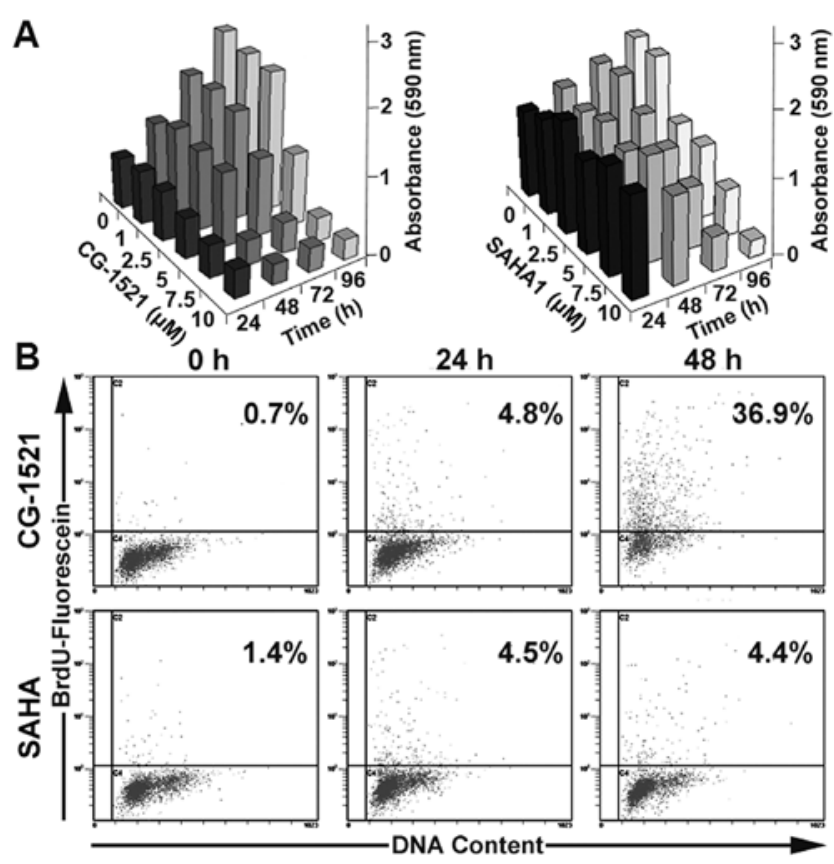

Figure 1. (A) Effect of CG-1521 and SAHA on the cell number and (B) apoptosis in MCF-7 cells. Cells were plated in 24 -well plates at $2 \times 10^{4}$ cels/well. After plating for $24 \mathrm{~h}$, cells were treated with 1-10 $\mu \mathrm{M}$ CG-1521 or SAHA. (A) The number of surviving cells at $24,48,72$, and $96 \mathrm{~h}$ was measured using the crystal violet assay. Data are representative of 3 independent experiments. Error bars are omitted for clarity. (B) MCF-7 cells were treated with $7.5 \mu \mathrm{M}$ CG-1521 or $7.5 \mu \mathrm{M}$ SAHA for 24 and $48 \mathrm{~h}$ as described above and DNA fragmentation was measured by Apo-BrdU labeling using flow cytometry as described in Materials and methods.

designed using Primer Express 1.5 (Applied Biosystems) and synthesized by Integrated DNA Technologies (Coraville, IA). Fold change of surveyed genes were analyzed using the $2^{-\Delta \Delta C T}$ method and calculated as a ratio to the houskeeping gene glyceraldehyde-3-phosphate dehydrogenase (GAPDH) (13). Expression levels are presented as mean \pm SEM of 3 independent experiments and considered statistically significant at $\mathrm{P} \leq 0.05$.

\section{Results}

$C G-1521$ induces apoptosis and $G_{0} / G_{1}$ arrest while SAHA induces apoptosis and $G_{2} / M$ arrest in MCF-7 cells. Crystal violet analysis shows a dose- and time-dependent decrease in cell number after treatment with CG-1521 or SAHA, indicative of apoptosis and/or cell cycle arrest (Fig. 1A). Both treatments decreased the cell number at concentrations above $5 \mu \mathrm{M}$ with CG-1521 inducing an effect as early as $48 \mathrm{~h}$. SAHA showed a decline in cell number at $72 \mathrm{~h}$. The decrease in cell number is due to the induction of apoptosis as measured by the increased DNA fragmentation assessed by Apo-BrdU labeling (Fig. 1B). CG-1521 induces significant levels of DNA fragmentation in MCF-7 cells by $48 \mathrm{~h}$, while DNA fragmentation induced by SAHA was considerably lower and did not increase significantly until $72 \mathrm{~h}$ (Fig. 1B and data not shown). These data suggest while both HDAC inhibitors induce cell death, CG-1521 elicits a much earlier cellular response than SAHA. To examine the underlying reason for this difference we assessed the effects of the 2 inhibitors on the expression of 

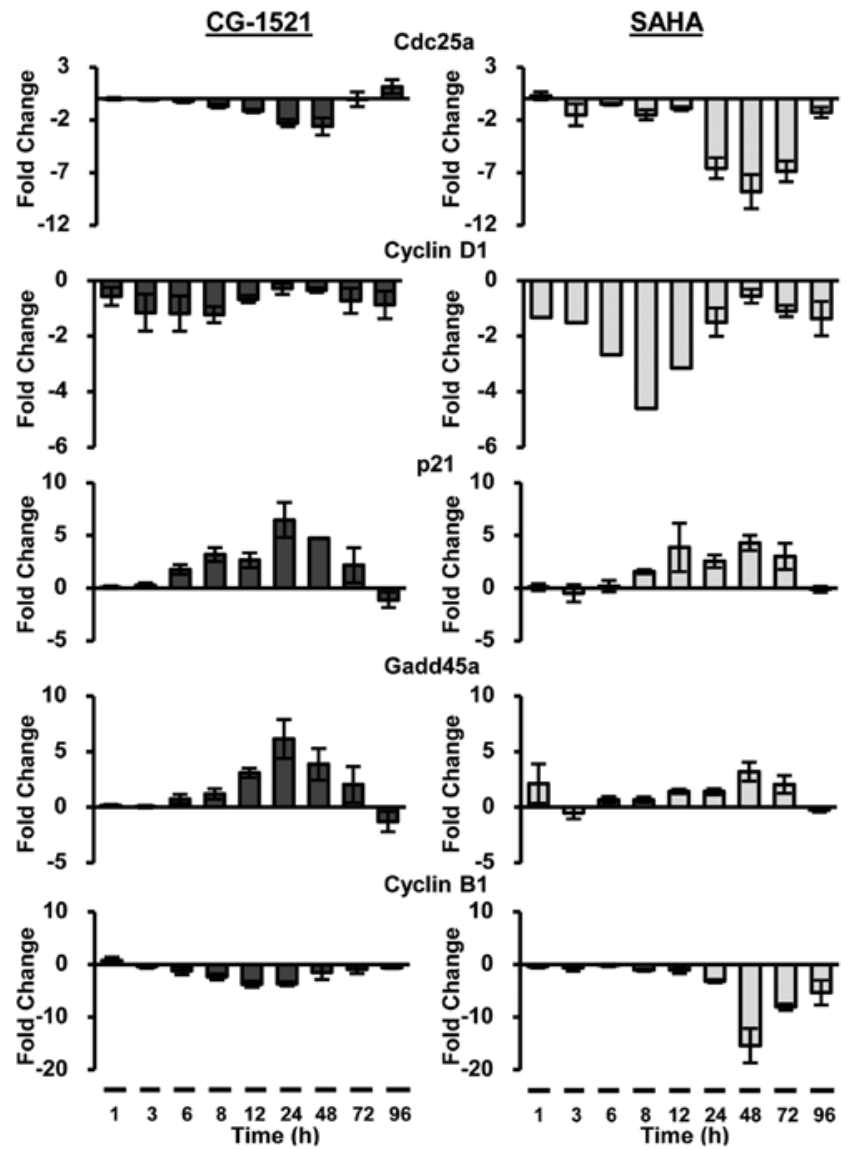

Figure 2. Changes in steady state mRNA levels of p53 target genes associated with the $\mathrm{G}_{1} / \mathrm{S}$ and the $\mathrm{G}_{2} / \mathrm{M}$ transition after treatment with $\mathrm{CG}-1521$ or SAHA. The steady state levels of cdc25a, Cyclin D1, p21, Gadd45a and Cyclin B1 mRNAs were assessed in MCF-7 cells treated with $7.5 \mu \mathrm{M}$ CG-1521 or $7.5 \mu \mathrm{M}$ SAHA from 0 to $96 \mathrm{~h}$. Results are expressed as mean $\pm \mathrm{SEM}$ of 3 independent experiments. Differences between means were considered statistically significant at $\mathrm{P} \leq 0.05$. Note scales on the $\mathrm{Y}$-axes vary from gene to gene.

several p53 target genes which have previously been shown to be induced by CG-1521 and to be associated with cell cycle arrest or apoptosis in other p53 wild type cell lines (10).

Real-time PCR of subsets of genes associated with $G_{I} / S$ arrest, $G_{2} / M$ arrest, and apoptosis/cell death

$\mathrm{G}_{l} / S$ transition associated genes (GO:0000082). To determine if the cellular responses to these drugs are due to differential gene induction, mRNA levels of 3 p53 target genes implicated in the $G_{1} / S$ transition, cdc25a, cyclin D1 and p21, were measured over a $96 \mathrm{~h}$ period (Fig. 2). The expression of cdc25a and cyclin D1 mRNA was significantly affected by treatment with SAHA. Cdc25a encodes a dual specificity phosphatase needed for $\mathrm{G}_{1} / \mathrm{S}$ transition and was downregulated, reaching a nadir at $48 \mathrm{~h}$, while the mRNA encoding cyclin D1 was downregulated more rapidly, reaching a nadir by $8 \mathrm{~h}$. In contrast CG-1521 treatment had minimal effect on the steady state level of these genes. The steady state mRNA level of $\mathrm{p} 21^{\text {Waf- } 1 / \text { cip- } 1}$ increased in MCF-7 cells after treatment with CG-1521, reaching a peak at $24 \mathrm{~h}$, while SAHA had less of an effect on $\mathrm{p} 21^{\text {Waf-1/cip-1 }}$ transcript levels.
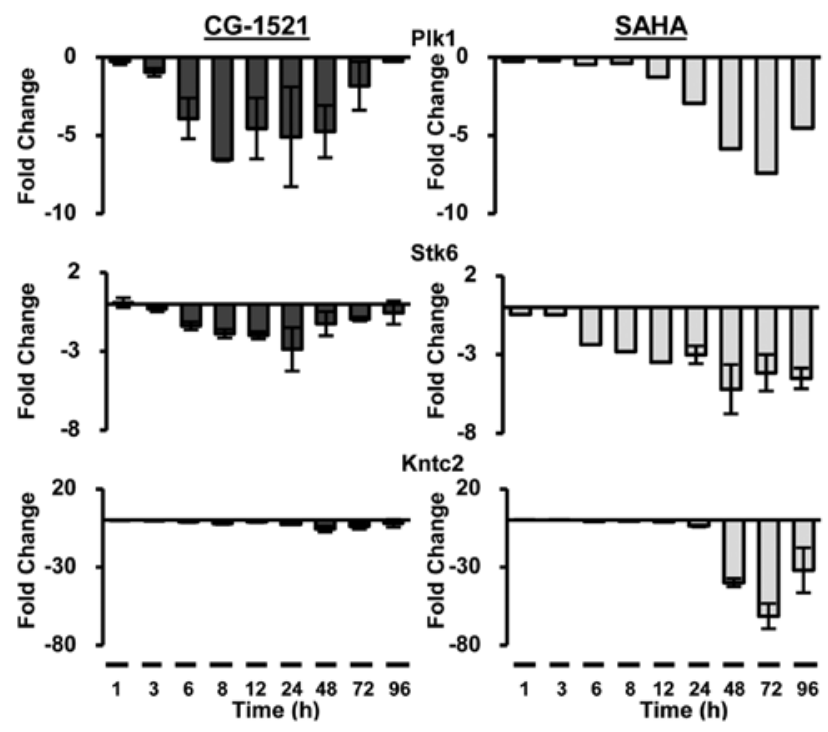

Figure 3. Changes in steady state mRNA levels of p53 target genes associated with spindle or kinetochore formation after treatment with CG-1521 or SAHA. The steady state levels of Plk1, Stk6 and Kntc2 mRNAs were assessed by qPCR in MCF-7 cells treated with $7.5 \mu \mathrm{M}$ CG-1521 or $7.5 \mu \mathrm{M}$ SAHA from 0 to $96 \mathrm{~h}$. Results are expressed as mean \pm SEM of 3 independent experiments. Differences between means were considered statistically significant at $\mathrm{P} \leq 0.05$. Note scales on the $\mathrm{Y}$-axes vary from gene to gene.

$G_{2} / M$ transition-associated genes (GO:0000086). $\mathrm{G}_{2} / \mathrm{M}$ transition associated genes were also surveyed to establish the effects of CG-1521 and SAHA on their expression (Fig. 2). The steady state mRNA level of Gadd45a (growth arrest and DNA-damage-inducible, $\alpha$ ) increased in MCF-7 cells after treatment with CG-1521, reaching a peak at $24 \mathrm{~h}$ while SAHA had minimal effects on the expression of this transcript. In contrast, SAHA significantly downregulated the expression of cyclin B1 $48 \mathrm{~h}$ after treatment while the downregulation of cyclin B1 in response to CG-1521 was much less pronounced than that seen with SAHA. Neither Wee1 nor Cks2 mRNA levels were affected by either of the treatments (data not shown).

Spindle and centrosome formation/biogenesis-associated genes (GO:0007051). Several p53 target genes are involved in spindle and centrosome formation during anaphase and spindle and centrosome biogenesis. A subset of these genes was also examined upon treatment with CG-1521 or SAHA (Fig. 3). Both CG-1521 and SAHA significantly downregulated Plk1 (polo like kinase 1) and Stk6 transcript levels, although CG-1521 appeared to downregulate the mRNA levels more rapidly that SAHA (6-12 h vs. 24-48 h). SAHA also considerably downregulated Kntc2 (the Ndc80 complex which modulates kinetochore assembly on microtubules) in this time frame, while CG-1521 did not affect the expression of Kntc2.

Cell death (GO:0008219) and apoptosis (GO:0006915)associated genes. Changes in the transcript levels of cell death and apoptosis-associated genes were examined after treatment with CG-1521 and SAHA (Fig. 4). Transcript levels of Bnip3 and its splice variant Bnip3L were upregulated with similar time courses after treatment with CG-1521, reaching a peak 

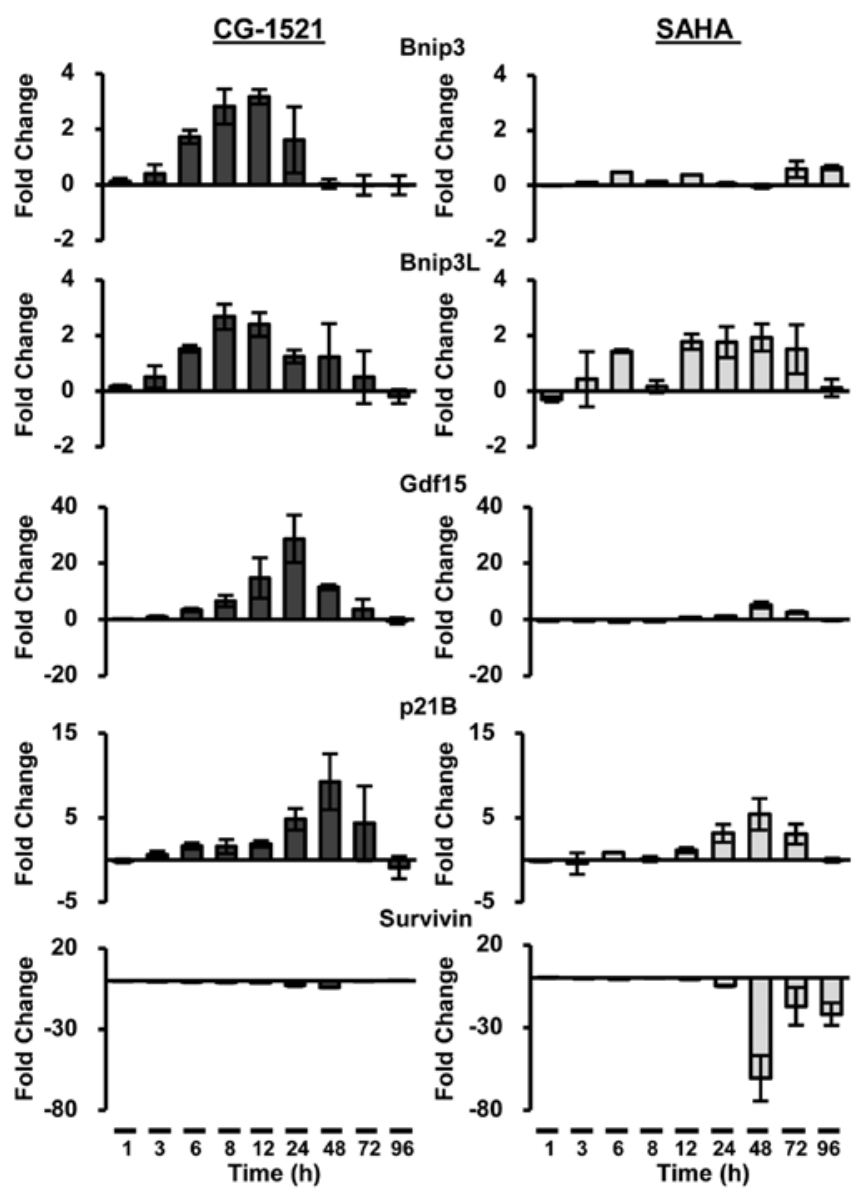

Figure 4. Changes in steady state mRNA levels of p53 target genes associated with cell death/apoptosis after treatment with CG-1521 or SAHA. The steady state levels of Bnip3, Bnip3L, Gdf15, p21B and survivin mRNAs were assessed in MCF-7 cells treated with $7.5 \mu \mathrm{M} \mathrm{CG}-1521$ or $7.5 \mu \mathrm{M}$ SAHA from 0 to $96 \mathrm{~h}$. Results are expressed as mean \pm SEM of 3 independent experiments. Differences between means were considered statistically significant at $\mathrm{P} \leq 0.05$. Note scales on the $\mathrm{Y}$-axes vary from gene to gene.

12-24 h after treatment. While the levels of Bnip3 did not change significantly after treatment with SAHA, Bnip3L transcript levels increased significantly after $24 \mathrm{~h}$ of treatment and remained elevated until 72 h. CG-1521 induced a substantial increase in the steady state levels of Gdf15 mRNA, which was upregulated as early as $6 \mathrm{~h}$ and peaked at $48 \mathrm{~h}$. In contrast SAHA did not significantly induce Gdf15 mRNA. The p21B splice variant that has been associated with apoptosis was upregulated $48 \mathrm{~h}$ after treatment by both CG-1521 and SAHA. SAHA also downregulated the transcript levels of the pro-survival gene survivin later in the experimental time course.

\section{Discussion}

The use of HDAC inhibitors as potential therapies for various cancers is gaining momentum as new research emerges regarding their ability to induce cell cycle arrest and apoptosis $(14,15)$. In this study we have characterized the response of MCF-7 breast cancer cells to two hydroxamic acid derivatives (CG-1521 and SAHA) that are considered to be pan-HDAC inhibitors. These studies demonstrate that both inhibitors influence cell cycle progression and cell death; however, they modulate gene expression with different time courses and different biological outcomes, suggesting that they inhibit individual HDACs with different efficiencies. CG-1521 induce genes associated with the $\mathrm{G}_{1} / \mathrm{S}$ transition (p21) and cell death (Bnip3, Bnip3L, Gdf15 and p21B), suggesting that the overriding effect of CG-1521 is to induce cell cycle arrest and apoptosis. In contrast, SAHA modulates the expression of Gadd45a and cyclin B1 and several p53 target genes associated with spindle assembly and cytokinesis [Plk1, Stk6 (Aurora kinase A) and Kntc2] suggesting that it predominantly blocks progression through the M-phase and may subsequently induce mitotic catastrophe and cell death (16).

Even though both CG-1521 and SAHA coordinate the catalytic site $\mathrm{Zn}^{2+}$ in Class I and Class II HDACs (17), it is apparent that the 2 compounds inhibit individual HDACs with different efficiencies, since they induce distinctive changes in gene expression and produce different biological endpoints. While the bulk of the enzyme activity of the HATs and HDACs is dedicated to modulating histone acetylation and chromatin conformation, it is now evident that the tumor suppressor p53 $(12,18)$ and many other transcription factors (19) are also acetylated and deacetylated by these enzymes. These posttranslational modifications influence the nuclear retention, protein binding partners and DNA binding activity of these transcription factors $(20,21)$. Recent studies have suggested that HDAC inhibitors may enhance the ability of p53 to bind to DNA (22) which may explain the temporal differences in gene expression induced by the 2 inhibitors. Alternatively it is possible that the metabolism of either CG-1521 or SAHA influences the timing of gene expression. Our previous studies suggested that CG-1521 and TSA stabilize different acetylated isoforms of p53 in prostate cancer cells, leading to the recruitment of different co-activator complexes, differences in gene expression and biological outcome (10-12). However, the present studies suggest that the biological response to HDAC inhibitors cannot be ascribed solely to the selective induction of gene expression by selective acetylated isoforms of p53. Similar comparative studies in lung cancer cell cultures have also demonstrated that HDAC inhibitors have overlapping gene targets (23).

Thus, while we have focused on the evaluation of the response of p53 target genes identified in our previous studies, it is likely that the transcription of other genes, influenced by the stabilization of acetylated histones in active chromatin or other acetylated transcription factors also influence the biological response of the cells to these inhibitors. Development of therapies for cancer or other diseases based on the use of HDAC inhibitors to modulate gene expression will require a full characterization of the effects of these inhibitors on the acetylation status of histone, non-histone and cytoplasmic proteins.

\section{Acknowledgements}

The authors would like to thank Errant Gene Therapeutics LLC, Chicago, IL, for the gift of CG-1521. A.K.K. and T.T. would like to acknowledge the support of the University of Notre Dame REU Summer Research Program. S.R. would like to acknowledge support from the Pat Girondi Post-Doctoral Fellowship in Molecular Medicine. 


\section{References}

1. Lane AA and Chabner BA: Histone deacetylase inhibitors in cancer therapy. J Clin Oncol 27: 5459-5468, 2009.

2. Selvi RB and Kundu TK: Reversible acetylation of chromatin: implication in regulation of gene expression, disease and therapeutics. Biotechnol J 4: 375-390, 2009.

3. Haberland M, Montgomery RL and Olson EN: The many roles of histone deacetylases in development and physiology: implications for disease and therapy. Nat Rev Genet 10: 32-42, 2009

4. Gregoretti IV, Lee YM and Goodson HV: Molecular evolution of the histone deacetylase family: functional implications of phylogenetic analysis. J Mol Biol 338: 17-31, 2004.

5. Yang XJ and Seto E: The Rpd3/Hda1 family of lysine deacetylases: from bacteria and yeast to mice and men. Nat Rev Mol Cell Biol 9: 206-218, 2008.

6. Haigis MC and Sinclair DA: Mammalian sirtuins: biological insights and disease relevance. Annu Rev Pathol 5: 253-295 2010.

7. Müller S and Krämer OH: Inhibitors of HDACs - effective drugs against cancer? Curr Cancer Drug Targets 10: 210-228, 2010

8. Neugebauer RC, Sippl W and Jung M: Inhibitors of NAD dependent histone deacetylases (sirtuins). Curr Pharm Des 14 562-573, 2008

9. Peck B, Chen CY, Ho KK et al: SIRT inhibitors induce cell death and p53 acetylation through targeting both SIRT1 and SIRT2. Mol Cancer Ther 9: 844-855, 2010.

10. Roy S, Jeffrey R and Tenniswood M: Array-based analysis of the effects of trichostatin A and CG-1521 on cell cycle and cell death in LNCaP prostate cancer cells. Mol Cancer Ther 7: 1931-1939, 2008.

11. Roy S, Packman K, Jeffrey R and Tenniswood M: Histone deacetylase inhibitors differentially stabilize acetylated p53 and induce cell cycle arrest or apoptosis in prostate cancer cells. Cell Death Differ 12: 482-491, 2005.
12. Roy S and Tenniswood M: Site-specific acetylation of p53 directs selective transcription complex assembly. J Biol Chem 282: 4765-4771, 2007.

13. Livak KJ and Schmittgen TD: Analysis of relative gene expression data using real-time quantitative PCR and the 2(-Delta Delta C(T)) method. Methods 25: 402-408, 2001

14. Venugopal B and Evans TR: Developing histone deacetylase inhibitors as anti-cancer therapeutics. Curr Med Chem 18: 1658-1671, 2011.

15. Marson CM: Histone deacetylase inhibitors: design, structureactivity relationships and therapeutic implications for cancer. Anticancer Agents Med Chem 9: 661-692, 2009.

16. Galluzzi L, Vitale I, Abrams JM et al: Molecular definitions of cell death subroutines: recommendations of the Nomenclature Committee on Cell Death 2012. Cell Death Differ: July 15, 2011 (Epub ahead of print). doi: 10.1038/cdd.2011.96, 2011.

17. Wang D: Computational studies on histone deacetylases and the design of selective histone deacetylase inhibitors. Curr Top Med Chem 9: 241-256 2009

18. Tang Y, Zhao W, Chen Y,Zhao Y, Gu W: Acetylation is indispensable for p53 activation. Cell 133: 612-626, 2008

19. Kim GW and Yang XJ: Comprehensive lysine acetylomes emerging from bacteria to humans. Trends Biochem Sci 36: 211-220, 2011.

20. Spange S, Wagner T, Heinzel T, and Krämer OH: Acetylation of non-histone proteins modulates cellular signalling at multiple levels. Int J Biochem Cell Biol 41: 185-198, 2009.

21. Buchwald M, Krämer $\mathrm{OH}$ and Heinzel T: HDACi-targets beyond chromatin. Cancer Lett 280: 160-167, 2009.

22. Harms KL and Chen X: Histone deacetylase 2 modulates p53 transcriptional activities through regulation of p53-DNA binding activity. Cancer Res 67: 3145-3152, 2007.

23. Zhang XH, Rao M, Loprieato JA, Hong JA et al: Aurora A, Aurora B and survivin are novel targets of transcriptional regulation by histone deacetylase inhibitors in non-small cell lung cancer. Cancer Biol Ther 7: 1388-1397, 2008. 